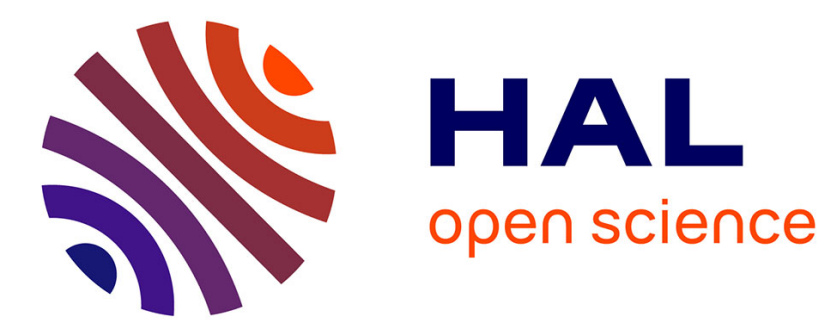

\title{
Games induced by the partitioning of a graph
}

Michel Grabisch, Alexandre Skoda

\section{To cite this version:}

Michel Grabisch, Alexandre Skoda. Games induced by the partitioning of a graph. Annals of Operations Research, 2012, 201 (1), pp.229-249. 10.1007/s10479-012-1200-8 . hal-00830291

\section{HAL Id: hal-00830291 https://hal.science/hal-00830291}

Submitted on 4 Jun 2013

HAL is a multi-disciplinary open access archive for the deposit and dissemination of scientific research documents, whether they are published or not. The documents may come from teaching and research institutions in France or abroad, or from public or private research centers.
L'archive ouverte pluridisciplinaire HAL, est destinée au dépôt et à la diffusion de documents scientifiques de niveau recherche, publiés ou non, émanant des établissements d'enseignement et de recherche français ou étrangers, des laboratoires publics ou privés. 


\title{
Games induced by the partitioning of a graph*
}

\author{
M. Grabisch ${ }^{\dagger} \quad$ A. Skoda ${ }^{\ddagger}$
}

March 26, 2012

\begin{abstract}
The paper aims at generalizing the notion of restricted game on a communication graph, introduced by Myerson. We consider communication graphs with weighted edges, and we define arbitrary ways of partitioning any subset of a graph, which we call correspondences. A particularly useful way to partition a graph is obtained by computing the strength of the graph. The strength of a graph is a measure introduced in graph theory to evaluate the resistance of networks under attacks, and it provides a natural partition of the graph (called the Gusfield correspondence) into resistant components. We perform a general study of the inheritance of superadditivity and convexity for the restricted game associated with a given correspondence. Our main result is to give for cycle-free graphs necessary and sufficient conditions for the inheritance of convexity of the restricted game associated with the Gusfield correspondence.
\end{abstract}

Keywords: communication networks, coalition structure, cooperative game, strength of a graph.

\section{Introduction}

Communication games were introduced by Myerson in 1977 [10]. These are cooperative games $(N, v)$ defined on the set of vertices $N$ of an undirected graph $G=(N, E)$, where $E$ is the set of edges. $v$ is the characteristic function of the game, $v: 2^{N} \rightarrow \mathbb{R}, A \mapsto v(A)$ and verifies $v(\emptyset)=0$. The graph $G$ describes how the players of $N$ can communicate: $e=\{i, j\} \in E$ if and only if the players $i$ and $j$ can directly communicate. For every coalition $A \subseteq N$, we consider the induced restricted graph $G_{A}:=(A, E(A))$, where

\footnotetext{
${ }^{*}$ The authors wish to thank an anonymous referee, whose comments permitted to improve greatly the presentation of the paper.

${ }^{\dagger}$ Paris School of Economics, Université de Paris I, 106-112 Bd de l'Hôpital, 75013 Paris, France. E-mail: michel.grabisch@univ-paris1.fr

${ }^{\ddagger}$ Corresponding author. Université de Paris I, Centre d'Economie de la Sorbonne, 106-112 Bd de l'Hôpital, 75013 Paris, France. E-mail: alexandre.skoda@univ-paris1.fr
} 
$E(A)$ is the set of edges $e=\{i, j\} \in E$ such that $i$ and $j$ are in $A$. We denote by $A / G$ the set of connected components of $G_{A}$, that is, those sets $F$ which are maximal subcoalitions of $A$ such that all pairs of players $i, j$ in $F$ can communicate by a path in $G_{A}$ starting from $i$ and ending at $j$. Myerson defined the network-restricted game $(N, \bar{v})$,

$$
\bar{v}(A)=\sum_{F \in A / G} v(F) \text {, for all } A \subseteq N .
$$

The new game $(N, \bar{v})$ takes into account how the players of $N$ can communicate according to the graph $G$. Owen [11] proved that if $(N, v)$ is superadditive then $(N, \bar{v})$ is also superadditive without any assumption on $G$.

The aim of the paper is to take a more general view, by noting that $A / G$ is a partition of $A$, and considering instead arbitrary ways of partitioning any subset of vertices. We call correspondence any way of partitioning, formally a mapping $\mathcal{P}$ on $2^{N}$, assigning to any nonempty $A \subseteq N$ a partition $\mathcal{P}(A)$ of $A$. Then the above formula becomes

$$
\bar{v}(A)=\sum_{F \in \mathcal{P}(A)} v(F) \text {, for all } A \subseteq N,
$$

which defines what we call the main restricted game associated with $\mathcal{P}$. Then natural questions arise: supposing that $v$ is superadditive, will $\bar{v}$ be superadditive too? What about convexity of $\bar{v}$ when $v$ is convex? These questions are important in cooperative game theory, since their answers condition the existence of the set of imputations, of the core, and whether the Shapley value lies in the core.

Of course, generality cannot be considered as an aim per se, and must convey some meaning in order to be useful. To this aim, we consider in this paper weighted graphs, where each edge $e \in E$ has a weight $u(e)$, whose interpretation may depend on the context (e.g., a degree of friendship, a level of communication, a resistance under attacks, or a security level, etc.). In this framework, an obvious way of partitioning a coalition $A \subseteq N$, which we denote by $\mathcal{P}_{\min }$, is to remove all edges of minimum weight in $A$. The reason to do this is that these edges are weak in some sense, and may easily disappear. The components of $\mathcal{P}(A)$ should then show the "stronger" components of the graph.

There is however a more clever way to formalize the notion of "strength" of a graph, which has been introduced by Gusfield [9] for graphs with edges of unit strength and generalized to arbitrary edge-strengths by Cunningham [4]. The strength $\sigma(G, u)$ of $G$ is defined by:

$$
\sigma(G, u):=\min _{A \subseteq E} \frac{u(A)}{k(A)-k(\emptyset)}
$$

where $k(A)$ is the number of connected components of the graph $G=$ $(N, E \backslash A)$ (and $k(\emptyset)$ the number of components of $G$ ). $\sigma$ can be seen 
as a measure of the resistance of the network $G$ under attack. Indeed, if we suppose that someone wants to destroy as much as possible the communication possibilities, and that the effort required to delete a link between two players is proportional to the strength of this link, then $\sigma(G, u)$ is the minimal average effort one has to make to augment the number of components as much as possible by deleting a subset $A$ of edges of $G$. The partition of $N$ in connected components corresponding to the graph $G=(N, E \backslash A)$, where $A$ is a minimizer in the definition of $\sigma$, provides a decomposition of $N$ into connected components which are strongly coherent in the following sense: they take into account both the strength $u(e)$ of the links of communication and the combinatorial structure of the communication graph $G$.

We denote by $\mathcal{P}_{G}$ the correspondence arising from the strength. The restricted game $\bar{v}$ associated with $\mathcal{P}_{G}$ reflects both the combinatorial structure of the graph, like the Myerson game, and the strength of the graph. We will give sufficient and necessary conditions for the inheritance of convexity from $v$ to $\bar{v}$ for cycle-free graphs, and this constitutes the main result of the paper (Theorem 12).

We propose also another kind of restricted game associated with $\mathcal{P}_{G}$, by in some sense iterating the processus of partitioning, that is, we partition by $\mathcal{P}_{G}$ each component of the graph partitioned by $\mathcal{P}_{G}$, and continue this process until the partition of $N$ in singletons is obtained. Then, for a given $A \subseteq N, \mathcal{P}(A)$ is built by taking the biggest possible components in the successive partitions. We denote by $\tilde{v}$ the restricted game associated with this partition, and show that convexity is inherited from $v$ to $\tilde{v}$ in any case, using results by Algaba et al. [1], and Faigle [5].

The article is organized as follows. We define in Section 2 the partitions associated with the strength of a graph. After having defined the game associated with a correspondence in Section 3, we establish necessary and sufficients conditions for inheritance of superadditivity in Section 4. We give a simple counterexample to inheritance of superadditivity (and therefore of convexity) in the general case. Then we define a slightly weaker condition than convexity in Section 5.1 and establish necessary conditions on the edgeweights to have inheritance of this property. Then we prove that these conditions are also sufficient in the case of cycle-free graphs for superadditive games. We compute the Shapley value of the game $(N, \bar{v})$ in the case of cyclefree graphs in Section 6. Finally we study in Section 7 the inheritance of superadditivity and convexity from $(N, v)$ to $(N, \tilde{v})$. 


\section{Partition associated with the strength of a graph}

Let $G=(N, E)$ be a connected graph and let $u: E \rightarrow \mathbb{R}^{+}$be a weight function on the set $E$ of edges. The strength of $G$ is defined by:

$$
\sigma(G, u):=\min _{A \subseteq E} \frac{u(A)}{k(A)-1}
$$

where $k(A)$ is the number of connected components of the graph $G=(N, E \backslash$ $A$ ). When $G$ and $u$ are fixed and that there is no ambiguity, we simply denote by $\sigma$ the strength $\sigma(G, u)$ of $G$. The computation of the strength is a polynomial problem [4].

Let $r$ be the rank function associated with $G$, i.e., for all $A \subseteq E, r(A)$ denotes the size of a maximal forest included in $A$. The rank function is submodular:

$$
r(A \cup B)+r(A \cap B) \leq r(A)+r(B) \quad \forall A, B \subseteq E .
$$

As $G$ is a connected graph, we have $r(E)=|V|-1$ and $r(E \backslash A)=|V|-k(A)$. Thus $k(A)-1=r(E)-r(E \backslash A)$ and:

$$
\sigma(G, u)=\min _{A \subseteq E} \frac{u(A)}{r(E)-r(\bar{A})} .
$$

We will define the strength of a not necessarily connected graph by this last formula because this last definition naturally extends to the case of a matroid or a polymatroid (cf $[7,6,13])$.

Let us define the auxiliary function $f$ by, $\forall A \subseteq E$ :

$$
\begin{aligned}
f(A) & :=u(A)-\sigma(k(A)-1) \\
& =u(A)+\sigma r(\bar{A})-\sigma r(E) .
\end{aligned}
$$

As $r$ is submodular and $u$ is additive, $f$ is submodular. By definition of $\sigma$, $\forall A \subseteq E, f(A) \geq 0$ and $f(A)=0$ with $A \neq \emptyset$ is equivalent to $\frac{u(A)}{k(A)-1}=\sigma$, that is, $A$ realizes the minimum of the strength. Thus $A \neq \emptyset$ realizes the minimum of the ratio in the definition of the strength if and only if $A$ realizes the minimum of the submodular function $f$. It is a classical result that the family of sets which realizes the minimum of a given submodular function is closed under union and intersection. Hence the family $\{A \subseteq E ; f(A)=0\}$ is closed under union and intersection, and there exists a maximal element $A_{\text {max }}$ in $\mathcal{F}$ and a minimal one $A_{\text {min }}$ in $\mathcal{F}$. If $A_{\text {min }} \neq \emptyset$, we have:

$$
\sigma=\frac{u\left(A_{\max }\right)}{k\left(A_{\max }\right)-1}=\frac{u\left(A_{\min }\right)}{k\left(A_{\min }\right)-1}
$$

and for all $A \subseteq E$ such that $\sigma=\frac{u(A)}{k(A)-1}$ we have:

$$
A_{\min } \subseteq A \subseteq A_{\max }
$$


For example, if the graph $G$ is a tree and if all weights are equal to 1 , every subset $A \neq \emptyset$ is a minimizer of the strength:

$$
\sigma=\frac{|A|}{k(A)-1}=\frac{|A|}{|A|}=1 .
$$

We have $A_{\max }=E$ and $A_{\min }=\emptyset$ and there are as many partitions of $N$ associated with the strength as nonempty subsets $A$ of $E$. Especially each edge $e$ is a minimal minimizer but there is no smallest minimizer.

\section{The main restricted game associated with a cor- respondence}

We now consider a correspondence $\mathcal{P}$ which associates to every subset $A \subseteq N$ a partition $\mathcal{P}(A)$ of $A$. Then for every game $(N, v)$ we define the main restricted game $(N, \bar{v})$ associated with $\mathcal{P}$ by:

$$
\bar{v}(A)=\sum_{F \in \mathcal{P}(A)} v(F), \text { for all } A \subseteq N .
$$

Thereafter we consider in particular two specific correspondences. We denote by $\mathcal{P}_{G}$ the correspondence which associates to every subset $A \subseteq N$ the partition associated with the strength of the graph $G_{A}=(A, E(A))$, and we refer to it as the Gusfield correspondence. We denote by $\sigma(A)$ the strength of $G_{A}$. As we have already noticed in Section 2, there may be several minimizers for the strength of a given graph and therefore several possible partitions. We will select the maximal subset $A_{\max }$ of $E(A)$ we can delete to achieve the minimum in the definition of the strength of $G_{A}$ (as defined in Section 2), and denote by $\mathcal{P}_{\max }(A)$ the corresponding partition. Therefore the main restricted game $(N, \bar{v})$ associated with $\mathcal{P}_{G}$ is defined by:

$$
\bar{v}(A)=\sum_{F \in \mathcal{P}_{\max }(A)} v(F), \text { for all } A \subseteq N .
$$

For a given subset $A \subseteq N$, we denote by $\Sigma(A)$ the subset of edges of minimum weight in $E(A)$, i.e., $e \in \Sigma(A)$ if and only if $u(e)=\min _{e^{\prime} \in E(A)} u\left(e^{\prime}\right)$. We denote by $\mathcal{P}_{\min }$ the correspondence which associates to every subset $A \subseteq N$ the partition $\mathcal{P}_{\min }(A)$ whose elements are the components of the graph $G_{A}=(A, E(A) \backslash \Sigma(A))$. For cycle-free graphs $\mathcal{P}_{G}$ coincides with $\mathcal{P}_{\text {min. }}$. In particular, for a given subset $A \subseteq N$, we have $A_{\max }=\Sigma(A)$ and the strength of $G_{A}$ satisfies $\sigma(A)=\min _{e^{\prime} \in E(A)} u\left(e^{\prime}\right)$. For example, if $G$ is a tree with all edge-weights equal to 1 , then for every subset $A \subseteq N$, $\mathcal{P}_{\max }(A)$ is the singletons partition of $A$ and $(N, \bar{v})$ is the trivial restricted game defined by:

$$
\bar{v}(A)=\sum_{i \in A} v(\{i\}), \text { for all } A \subseteq N .
$$


In the following sections, we are going to study conditions on partitions and on the edge-weights to have inheritance of superadditivity and of convexity. Some of the results are only valid for cycle-free graphs and we will then refer to $\mathcal{P}_{\min }$.

\section{Inheritance of superadditivity}

We first establish necessary and sufficient conditions for inheritance of superadditivity. We recall that a game $(N, v)$ is superadditive if, for all $A, B \in 2^{N}$ such that $A \cap B=\emptyset, v(A \cup B) \geq v(A)+v(B)$. For any given subset $\emptyset \neq S \subseteq N$, the unanimity game $\left(N, u_{S}\right)$ is defined by:

$$
u_{S}(A)= \begin{cases}1 & \text { if } A \supseteq S \\ 0 & \text { otherwise }\end{cases}
$$

Theorem 1. Let $G=(N, E, u)$ be an arbitrary weighted graph and $\mathcal{P}$ be an arbitrary correspondence on $N$. Then the following claims are equivalent:

1) For all $\emptyset \neq S \subseteq N, \overline{u_{S}}$ is superadditive.

2) For all $\emptyset \neq S \subseteq N$, $\overline{u_{S}}$ is nondecreasing.

3) For all subsets $A \subseteq B \subseteq N, \mathcal{P}(A)$ is a refinement of the restriction of $\mathcal{P}(B)$ to $A$.

4) For all superadditive game $(N, v)$ the restricted game $(N, \bar{v})$ is superadditive.

Proof. A superadditive and non-negative function is obviously non-decreasing, therefore 1) implies 2). Let us now suppose 2) is satisfied and let us consider $A \subseteq B \subseteq N$. Let us denote by $\mathcal{P}(B)=\left\{B_{1}, B_{2}, \ldots, B_{m}\right\}$ the partition of $B$. For all $A_{l} \in \mathcal{P}(A)$, we have:

$$
1=\overline{u_{A_{l}}}(A) \leq \overline{u_{A_{l}}}(B) .
$$

But $\overline{u_{A_{l}}}$ only takes values 0 or 1 , therefore we have equality in (12). As $\overline{u_{A_{l}}}(B)=\sum_{j=1}^{m} u_{A_{l}}\left(B_{j}\right)$, there exists a unique element $B_{j}$ of $\mathcal{P}(B)$ such that $A_{l} \subseteq B_{j}$. Therefore 2) implies 3). Let us now suppose 3) is satisfied. Let us consider a superadditive game $(N, v)$ and $A, B \subseteq N$ such that $A \cap B=\emptyset$. Then we have:

$$
\bar{v}(A \cup B)=\sum_{C \in \mathcal{P}(A \cup B)} v(C)=\sum_{C \in \mathcal{P}(A \cup B)} v((C \cap A) \cup(C \cap B)) .
$$

As $C \cap A$ and $C \cap B$ are disjoint and $v$ is superadditive, (13) implies:

$$
\bar{v}(A \cup B) \geq \sum_{C \in \mathcal{P}(A \cup B)}(v(C \cap A)+v(C \cap B)) .
$$


As $\mathcal{P}(A)$ (resp. $\mathcal{P}(B)$ ) is a refinement of $\mathcal{P}(A \cup B$ ) restricted to $A$ (resp. $B$ ), for every $C \in \mathcal{P}(A \cup B)$ such that $C \cap A \neq \emptyset$ (resp. $C \cap B \neq \emptyset), C \cap A$ (resp. $C \cap B$ ) is a disjoint union of blocks of $\mathcal{P}(A)$ (resp. $\mathcal{P}(B)$ ). As $v$ is superadditive, we obtain:

$$
\bar{v}(A \cup B) \geq \sum_{C \in \mathcal{P}(A \cup B)}\left[\sum_{F \subseteq C \cap A, F \in \mathcal{P}(A)} v(F)+\sum_{F \subseteq C \cap B, F \in \mathcal{P}(B)} v(F)\right]
$$

which yields:

$$
\bar{v}(A \cup B) \geq \sum_{F \in \mathcal{P}(A)} v(F)+\sum_{F \in \mathcal{P}(B)} v(F)=\bar{v}(A)+\bar{v}(B) .
$$

Therefore 3 ) implies 4). Finally, as 4) trivially implies 1 ), we have equivalence of every claims.

Then Theorem 1 implies the following result.

Corollary 2. Let $G=(N, E, u)$ be an arbitrary weighted graph. If we consider on $G$ the correspondence $\mathcal{P}_{\min }$, then for every superadditive game $(N, v)$, the restricted game $(N, \bar{v})$ is superadditive.

Proof. Let us consider $A \subseteq B \subseteq N$. We have either $\Sigma(B) \cap E(A)=\Sigma(A)$ or $\Sigma(B) \cap E(A)=\emptyset . \mathcal{P}(A)$ is the partition of $A$ corresponding to the components of the subgraph $(A, E(A) \backslash \Sigma(A))$. If two elements of $A$ are connected by a path $\gamma$ in the subgraph $(A, E(A) \backslash \Sigma(A))$, then they are also connected by $\gamma$ in the subgraph $(B, E(B) \backslash \Sigma(B))$. Therefore $\mathcal{P}(A)$ is a refinement of the restriction of $\mathcal{P}(B)$ to $A$ and the result is a consequence of Theorem 1.

If we consider the Gusfield correspondence $\mathcal{P}_{G}$, the graph represented in Figure 1 shows that we do not have inheritance of the superadditivity in general, even if all weights are equal. Taking $A=\{1,2,3,4\}$ and $B=$

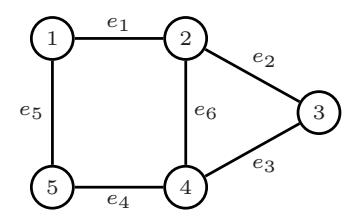

Figure 1:

$\{1,2,3,4,5\}$, we get $\mathcal{P}_{\max }(B)=\{\{1\},\{2\},\{3\},\{4\},\{5\}\}$ and $\mathcal{P}_{\max }(A)=$ $\{\{1\},\{2,3,4\}\}$. Therefore $\mathcal{P}_{\max }(A)$ is not a refinement of the restriction of $\mathcal{P}_{\max }(B)$ to $A$, and Theorem 1 proves that there is no inheritance of the superadditivity.

We end this section with an immediate consequence of Theorem 1.

Corollary 3. If there is inheritance of convexity for all unanimity games, then there is inheritance of superadditivity for all superadditive games. 


\section{Inheritance of convexity}

\subsection{Necessary conditions}

In this part we establish necessary conditions on the weight vector $u$ for the inheritance of convexity from the original communication game $(N, v)$ to the restricted game $(N, \bar{v})$. Actually, we are going to establish necessary conditions for a slightly weaker condition than convexity. Let $\mathcal{F}$ be a weakly union-closed family ${ }^{1}$ of subsets of $N$ such that $\emptyset \notin \mathcal{F}$. A game $v$ on $2^{N}$ is said to be $\mathcal{F}$-convex if for all $A, B \in \mathcal{F}$ such that $A \cap B \in \mathcal{F}$, we have:

$$
v(A \cup B)+v(A \cap B) \geq v(A)+v(B) .
$$

Of course convexity implies $\mathcal{F}$-convexity. The $\mathcal{F}$-convexity implies also the following condition. If a game $v$ on $2^{N}$ is $\mathcal{F}$-convex then, for all $i \in N$ and all $A \subseteq B \subseteq N \backslash\{i\}$ such that $A, B$ and $A \cup\{i\} \in \mathcal{F}$ we have:

$$
v(B \cup\{i\})-v(B) \geq v(A \cup\{i\})-v(A) .
$$

We say that a subset $A \subseteq N$ is connected if the induced graph $G_{A}=$ $(A, E(A))$ is connected. The family of connected subsets of $N$ is obviously weakly union-closed. We first establish that for this last family we have equivalence of these two conditions.

Theorem 4. Let $G=(N, E)$ be an arbitrary graph and let $\mathcal{F}$ be the family of connected subsets of $N$. Then the following conditions are equivalent:

$$
\begin{aligned}
& v(B \cup\{i\})-v(B) \geq v(A \cup\{i\})-v(A), \forall i \in N, \forall A, B \in \mathcal{F} \text { s.t. } \\
& A \subseteq B \subseteq N \backslash\{i\} \text { and } A \cup\{i\} \in \mathcal{F} .
\end{aligned}
$$

The result is well known if $\mathcal{F}=2^{N}$. The proof is the same as Schrijver's [12] (p. 767) with minor changes (as we are dealing with connected subsets of $N$ ). At first we have to prove the following lemma.

Lemma 5. Let $G=(N, E)$ be an arbitrary graph. Let $S \subset T$ be two connected subsets of $N$. Then there exists a node $t \in T \backslash S$ such that $T \backslash\{t\}$ is still connected.

Proof. Let $T^{\prime}$ (resp. $S^{\prime}$ ) be a spanning tree of $G_{T}$ (resp. $G_{S}$ ). If $t$ is a leaf node of $T^{\prime}$ then $T^{\prime} \backslash\{t\}$ is a spanning tree of $G_{T \backslash\{t\}}$. Therefore if one of the leaf nodes of $T^{\prime}$ belongs to $T \backslash S$, the result follows. Otherwise all leaf nodes of $T^{\prime}$ are in $S$ and for all $t \in T \backslash S, T^{\prime} \backslash\{t\}$ is disconnected. But $T^{\prime} \backslash\{t\} \cup S^{\prime}$ is a spanning connected subgraph of $G_{T \backslash\{t\}}$.

\footnotetext{
${ }^{1} \mathcal{F}$ is weakly union-closed if $A \cup B \in \mathcal{F}$ for all $A, B \in \mathcal{F}$ such that $A \cap B \neq \emptyset$.
} 
Proof of Theorem 4. We assume (20) is satisfied and we establish (19) by induction on $|A \Delta B|$. If $|A \Delta B|=1$, then we have $A \subseteq B$ or $B \subseteq A$ and (19) is trivially satisfied. If $|A \Delta B|=2$, we may suppose $|A \backslash B|=1$ and $|B \backslash A|=1$ (otherwise $A \subseteq B$ or $B \subseteq A$ and (19) is trivial). Setting $S:=A \cap B, T:=A$ and $B \backslash A=\{i\}$, we have $S, T \in \mathcal{F}$ with $S \subseteq T$, and $S \cup\{i\} \in \mathcal{F}$. Then (19) is equivalent to $v(T \cup\{i\})+v(S) \geq v(T)+v(S \cup\{i\})$, which is equivalent to (20) applied to $S$ and $T$ and therefore (19) is satisfied. If now $|A \Delta B| \geq 3$, we may assume by symmetry of $A$ and $B$ that $|B \backslash A| \geq 2$. Applying Lemma 5, we can find $t \in B \backslash A$ such that $B \backslash\{t\}$ is still connected. By induction, we apply (19) to the pair $\{A, B \backslash\{t\}\}$ :

$$
v(A \cup(B \backslash\{t\}))-v(B \backslash\{t\}) \geq v(A)-v(A \cap B)
$$

because $|A \Delta(B \backslash\{t\})|=|A \Delta B|-1<|A \Delta B|$. By induction we now apply (19) to the pair $\{A \cup(B \backslash\{t\}), B\}$ :

$$
v(A \cup(B \backslash\{t\}) \cup B)-v(B) \geq v(A \cup(B \backslash\{t\}))-v(B \backslash\{t\})
$$

because $|(A \cup(B \backslash\{t\})) \Delta B|=|A \backslash B|+1<|A \backslash B|+|B \backslash A|=|A \Delta B|$. Let us observe that $A \cup(B \backslash\{t\})$ is connected because $A$ and $B \backslash\{t\}$ are connected and their intersection is $A \cap B \neq \emptyset$. As $A \cup(B \backslash\{t\}) \cup B=A \cup B$, (22) may be written as:

$$
v(A \cup B)-v(B) \geq v(A \cup(B \backslash\{t\}))-v(B \backslash\{t\}) .
$$

(21) and (23) imply $v(A \cup B)-v(B) \geq v(A)-v(A \cap B)$.

Proposition 6. Let $G=(N, E, u)$ be an arbitrary weighted graph and $\mathcal{P}$ be an arbitrary correspondence on $N$. If for all non-empty subset $S \subseteq N, \overline{u_{S}}$ is superadditive then for all $A, B \subseteq N$ the following claims are satisfied.

1) Each element of $\mathcal{P}(A \cup B)$ is a finite union of elements of $\mathcal{P}(A)$ and $\mathcal{P}(B)$.

2) If $A_{j} \in \mathcal{P}(A), B_{k} \in \mathcal{P}(B)$ and $A_{j} \cap B_{k} \neq \emptyset$ then $A_{j}$ and $B_{k}$ are subsets of the same element of $\mathcal{P}(A \cup B)$.

Proof. As for all non-empty $S \subseteq N, \overline{u_{S}}$ is superadditive, Theorem 1 implies that $\mathcal{P}(A)$ (resp. $\mathcal{P}(B))$ is a refinement of $\mathcal{P}(A \cup B)_{\mid A}$ (resp. $\left.\mathcal{P}(A \cup B)_{\mid B}\right)$. That is each $A_{j} \in \mathcal{P}(A)$ (resp. $B_{k} \in \mathcal{P}(B)$ ) is a subset of some component $D_{j}$ (resp. $\left.D_{k}\right)$ in $\mathcal{P}(A \cup B)$. As $A \cup B=\left(\cup_{j} A_{j}\right) \cup\left(\cup_{k} B_{k}\right)$, each $D_{l} \in \mathcal{P}(A \cup B)$ is a finite union of such $A_{j}$ 's and $B_{k}$ 's. 2) is an obvious consequence of 1 ) as $\mathcal{P}(A \cup B)$ is a partition.

Lemma 7. Let $G=(N, E, u)$ be an arbitrary weighted graph, and $\mathcal{P}$ an arbitrary correspondence on $N$. Let us consider $A, B \subseteq N$ such that $A \cap B \neq$ $\emptyset$. If for all non-empty subset $S \subseteq N, \overline{u_{S}}$ is superadditive, then the following claims are equivalent. 
1) For all $\emptyset \neq S \subseteq N$, we have :

$$
\overline{u_{S}}(A \cup B)+\overline{u_{S}}(A \cap B) \geq \overline{u_{S}}(A)+\overline{u_{S}}(B) .
$$

2) $\mathcal{P}(A \cap B)=\left\{A_{j} \cap B_{k} ; A_{j} \in \mathcal{P}(A), B_{k} \in \mathcal{P}(B), A_{j} \cap B_{k} \neq \emptyset\right\}$.

Proof. We first prove that 1) implies 2). As $A \cap B$ is a subset of $A$ and $B$, Theorem 1 implies that $\mathcal{P}(A \cap B)$ is a refinement of $\mathcal{P}(A)_{\mid A \cap B}$ and $\mathcal{P}(B)_{\mid A \cap B}$. Hence, for every $C_{l} \in \mathcal{P}(A \cap B)$, there exists $A_{j} \in \mathcal{P}(A)$ and $B_{k} \in \mathcal{P}(B)$ such that $C_{l} \subseteq A_{j} \cap B_{k}$. Taking $\left.S=A_{j} \cap B_{k}, 1\right)$ implies $\overline{u_{S}}(A \cup B)+\overline{u_{S}}(A \cap B) \geq$ $\overline{u_{S}}(A)+\overline{u_{S}}(B)=1+1=2$. As $\overline{u_{S}}$ only takes values 0 or 1 , the last inequality implies $\overline{u_{S}}(A \cup B)=\overline{u_{S}}(A \cap B)=1$. Therefore there exists $C_{p} \in \mathcal{P}(A \cap B)$ such that $A_{j} \cap B_{k} \subseteq C_{p}$. As $\mathcal{P}(A \cap B)$ is a partition, we must have $C_{l}=C_{p}$ and therefore $C_{l}=A_{j} \cap B_{k}$.

We now prove 2) implies 1). If $\overline{u_{S}}(A)=\overline{u_{S}}(B)=0$, then (24) is trivially satisfied. Let us now assume w.l.o.g. $\overline{u_{S}}(A)=1$. Then there exists $A_{j} \in$ $\mathcal{P}(A)$ such that $S \subseteq A_{j}$. As $A \subseteq A \cup B$, Theorem 1 implies that $\mathcal{P}(A)$ is a refinement of $\mathcal{P}(A \cup B)_{\mid A}$, i.e., there exists $D_{j} \in \mathcal{P}(A \cup B)$ such that $A_{j} \subseteq D_{j}$ and therefore $\overline{u_{S}}(A \cup B)=1$. If $\overline{u_{S}}(B)=0$, then (24) is satisfied. If $\overline{u_{S}}(B)=1$, there exists $B_{k} \in \mathcal{P}(B)$ such that $S \subseteq B_{k}$. Hence $S \subseteq A_{j} \cap B_{k}$ and 2) implies $A_{j} \cap B_{k} \in \mathcal{P}(A \cap B)$, therefore $\overline{u_{S}}(A \cap B)=1$ and (24) is still satisfied.

Theorem 8. Let $G=(N, E, u)$ be an arbitrary weighted graph, $\mathcal{P}$ an arbitrary correspondence on $N$, and $\mathcal{F}$ a weakly-union closed family of subsets of $N$ such that $\emptyset \notin \mathcal{F}$. If for each non-empty subset $S \subseteq N, \overline{u_{S}}$ is superadditive, then the following claims are equivalent.

1) For all non-empty subset $S \subseteq N$, the game $\left(N, \overline{u_{S}}\right)$ is $\mathcal{F}$-convex.

2) For all $A, B \in \mathcal{F}$ such that $A \cap B \in \mathcal{F}, \mathcal{P}(A \cap B)=\left\{A_{j} \cap B_{k} ; A_{j} \in\right.$ $\left.\mathcal{P}(A), B_{k} \in \mathcal{P}(B), A_{j} \cap B_{k} \neq \emptyset\right\}$.

Moreover if $\mathcal{F}=\{A \subseteq N ; A$ connected $\}$ then 1) and 2) are equivalent to:

3) For all $i \in N$ and for all $A, B \in \mathcal{F}, A \subseteq B \subseteq N \backslash\{i\}$ such that $A \cup\{i\} \in$ $\mathcal{F}$, we have for all $A^{\prime} \in \mathcal{P}(A \cup\{i\}), \mathcal{P}(A)_{\mid A^{\prime}}=\mathcal{P}(B)_{\mid A^{\prime}}$.

Proof. If $A \cap B \in \mathcal{F}$ then $A \cap B \neq \emptyset$. Applying Lemma 7, we have equivalence of 1) and 2). Let us suppose 2) is satisfied and let us consider $A \subseteq B \subseteq N \backslash$ $\{i\}$. As $(A \cup\{i\}) \cap B=A$, we have $\mathcal{P}(A)=\left\{A_{j} \cap B_{k} ; A_{j} \in \mathcal{P}(A \cup\{i\}), B_{k} \in\right.$ $\left.\mathcal{P}(B), A_{j} \cap B_{k} \neq \emptyset\right\}$ and it implies 3). Let us now suppose 3) is satisfied. Applying Lemma 7 to the pair $\{A \cup\{i\}, B\}$, we get $\overline{u_{S}}(B \cup\{i\})-\overline{u_{S}}(B) \geq$ $\overline{u_{S}}(A \cup\{i\})-\overline{u_{S}}(A), \forall i \in N, \forall A, B \in \mathcal{F}$ s.t. $A \subseteq B \subseteq N \backslash\{i\}$ and $A \cup\{i\} \in$ $\mathcal{F}$. Therefore if $\mathcal{F}=\{A \subseteq N ; A$ connected $\}$, then Theorem 4 implies 1). 
Let $\gamma=\left(e_{1}, e_{2} \ldots, e_{m}\right)$ be an induced elementary path of $G$ (i.e. a path with no repeated vertices which is an induced subgraph of $G$ ) with $e_{i}=\{i, i+1\}$ for $1 \leq i \leq m$. We denote by $u_{j}$ the weight of $e_{j}$.

Proposition 9. Let $G=(N, E, u)$ be an arbitrary weighted graph, and $\mathcal{F}$ the family of connected subsets of $N$. Let us consider the correspondence $\mathcal{P}_{\text {min }}$ or $\mathcal{P}_{G}$. If for every unanimity game $\left(N, u_{S}\right)$, the restricted game $\left(N, \overline{u_{S}}\right)$ is $\mathcal{F}$-convex, then for all induced elementary paths $\gamma=\left(e_{1}, e_{2} \ldots, e_{m}\right)$ in $G$, and for all $i, j, k$ such that $1 \leq i<j<k \leq m$, the edge-weights satisfy:

$$
u_{j} \leq \max \left(u_{i}, u_{k}\right) .
$$

Thus we have a property of convexity on the edge-weights along every induced elementary path in $G$.

Proof. Suppose there exists $i, j, k$ such that $1 \leq i<j<k \leq m$ and $u_{j}>$ $\max \left(u_{i}, u_{k}\right)$. At first we make several reductions of this situation. We can fix $e_{i}$ and $e_{k}$ and select an edge $e_{j}$ such that $u_{j}$ is maximal for all $e_{j}$ between $e_{i}$ and $e_{k}$. We now fix such an $e_{j}$ and we select a maximal index $i$ such that $i<j$ and $u_{i}<u_{j}$. In the same way, we select $k$ minimal such that $j<k$ and $u_{k}<u_{j}$. For all $\left.l \in\right] i, k$ [ we now have by construction $u_{i}<u_{l}=u_{j}$ and $u_{k}<u_{l}=u_{j}$. We can now shrink the path $\gamma$ to its restriction from $i$ to $k+1$ and suppose that $i=1$ and $k=m$. If necessary we can also exchange $\gamma$ with the inverse path starting from $m+1$ and ending at 1 to have $u_{1} \geq u_{m}$. Therefore we can suppose:

$$
\forall l, j \in] 1, m\left[, u_{l}=u_{j}>u_{1} \geq u_{m} .\right.
$$

We define the sets $A=\{2,3, \ldots, m\}, B=\{1,2, \ldots, m\}$ as represented in Figure 2 and we denote now by $i$ the vertex $m+1$. We have $A \subset B \subset$ $N \backslash\{i\}$ and $A, B$ and $A \cup\{i\}$ are connected. For every $S \subseteq N, \mathcal{P}(S)$ is

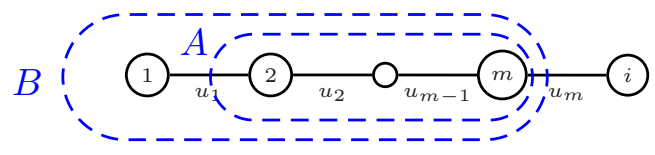

Figure 2:

obtained by deleting the edges with minimum weight in $E(S)$. Therefore, as a consequence of (26), we have $\mathcal{P}(A \cup\{i\})=\{A,\{i\}\}, \mathcal{P}(B)=\{A,\{1\}\}$, and $\mathcal{P}(A)=\{\{2\},\{3\}, \ldots,\{m\}\}$. Then $A \in \mathcal{P}(A \cup\{i\})$ but $\mathcal{P}(B)_{\mid A}=A \neq \mathcal{P}(A)$ and this contradicts Theorem 8 .

Remark 1. If $u_{1}$ (resp. $u_{m}$ ) is the smallest weight of the edges of $\gamma$, then the condition of convexity of the $u_{i}$ 's means that the sequence $\left(u_{i}\right)_{i=1}^{m}$ is non-decreasing (resp. non-increasing) as $u_{i} \leq \max \left(u_{1}, u_{i+1}\right)=u_{i+1}$ (resp. $\left.u_{i+1} \leq \max \left(u_{i}, u_{m}\right)=u_{i}\right)$ for all $1 \leq i \leq m-1$. 
Remark 2. We cannot restrict the convexity condition to only every 3uple of consecutive edges $u_{i} \leq \max \left(u_{i-1}, u_{i+1}\right), 2 \leq i \leq m-1$, because of the obvious counter-example: $u_{2}=u_{3}>\max \left(u_{1}, u_{4}\right)$. Nevertheless $u_{2}=$ $\max \left(u_{1}, u_{3}\right)$ and $u_{3}=\max \left(u_{2}, u_{4}\right)$.

Now we show there exists another necessary $\mathcal{F}$-convexity condition associated with every induced subgraph $(A, E(A))$ of $G$ corresponding to a star. A star $S_{k}$ corresponds to a tree with one internal node and $k$ leaves. We establish the result for stars with three leaves. The generalization to stars of greater size is immediate. We consider a star $S_{3}$ with vertices $\{1,2,3,4\}$ and edges $e_{1}=\{1,2\}, e_{2}=\{1,3\}$ and $e_{3}=\{1,4\}$.

Proposition 10. Let $G=(N, E, u)$ be an arbitrary weighted graph, and $\mathcal{F}$ the family of connected subsets of $N$. Let us consider the correspondence $\mathcal{P}_{\min }$ or $\mathcal{P}_{G}$. If for every unanimity game $\left(N, u_{S}\right)$, the restricted game $\left(N, \overline{u_{S}}\right)$ is $\mathcal{F}$-convex, then for every induced star of type $S_{3}$ of $G$, the edgeweights $u_{1}, u_{2}, u_{3}$ satisfy, after renumbering the edges if necessary:

$$
u_{1} \leq u_{2}=u_{3} .
$$

Proof. We have to prove that we cannot have two edge-weights strictly smaller than a third one. By contradiction let us assume we have $u_{1} \leq u_{2}<$ $u_{3}$, after renumbering if necessary. Let us consider the situation of Figure 3

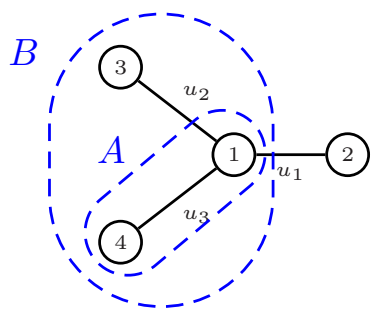

Figure 3:

where $A=\{1,4\}, B=\{1,3,4\}$, and $i=2$. By deleting the edge of minimal weight we obtain successively: $\mathcal{P}(B)=\{A,\{3\}\}, \mathcal{P}(A \cup\{i\})=\{A,\{i\}\}$ and $\mathcal{P}(A)=\{\{1\},\{4\}\}$. Therefore $A \in \mathcal{P}(A \cup\{i\})$ but $\mathcal{P}(B)_{\mid A}=A \neq \mathcal{P}(A)$ and it contradicts Theorem 8 .

Remark 3. For an induced star with $n$ edges $e_{1}, e_{2}, \ldots, e_{n}$ the weights verify $u_{1} \leq u_{2}=u_{3}=\cdots=u_{n}$ after renumbering the edges if necessary.

We can easily obtain necessary conditions for inheritance of convexity in the case of an induced cycle in $G$ on $m$ vertices with $m \geq 4$. Denote by $1,2, \ldots, m$ the nodes of an induced cycle $C$ and by $e_{1}, e_{2}, \ldots, e_{m}$ the edges with $e_{i}=\{i, i+1\}$ for $1 \leq i \leq m-1$ and $e_{m}=\{1, m\}$.

If $m=3$, it is easy to see that for every choice of the weights $u_{1}, u_{2}, u_{3}$ we have conservation of the convexity. Let $N=\{1,2,3\}, i=3$, and consider 
$\emptyset \neq A \subseteq B \subseteq N \backslash\{i\}$. If $A=B$ then $\bar{v}(B \cup\{i\})-\bar{v}(B)=\bar{v}(A \cup\{i\})-\bar{v}(A)$. If $A \subset B$, we can suppose $A=\{2\}$ and $B=\{1,2\}$ as represented in Figure 4. Then $\bar{v}(A)=v(2), \bar{v}(A \cup\{i\})=v(2)+v(i)$ and $\bar{v}(B)=v(1)+v(2)$. If

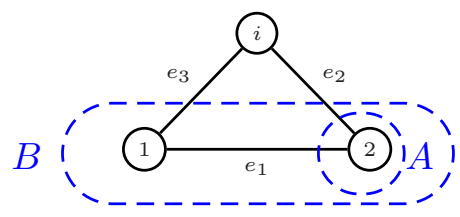

Figure 4:

$\bar{v}(B \cup\{i\})=v(B)+v(i)$ then $\bar{v}(B \cup\{i\})-\bar{v}(B)=v(\{1,2\})+v(i)-v(1)-v(2)$. If $\bar{v}(B \cup\{i\})=v(\{1, i\})+v(2)$ then $\bar{v}(B \cup\{i\})-\bar{v}(B)=v(\{1, i\})-v(1)$. As $v$ is supermodular, we have in these two cases $\bar{v}(B \cup\{i\})-\bar{v}(B) \geq v(i)$. If $\bar{v}(B \cup\{i\})=v(1)+v(2)+v(i)$ then $\bar{v}(B \cup\{i\})-\bar{v}(B)=v(i)$. Therefore in all cases we have $\bar{v}(B \cup\{i\})-\bar{v}(B) \geq \bar{v}(A \cup\{i\})-\bar{v}(A)$.

For $m \geq 4$ the inheritance of convexity implies strong restrictions on the edge-weights.

Proposition 11. Let $G=(N, E, u)$ be an arbitrary weighted graph, and $\mathcal{F}$ the family of connected subsets of $N$. Let us consider the correspondence $\mathcal{P}_{\min }$ or $\mathcal{P}_{G}$. If for every unanimity game $\left(N, u_{S}\right)$, the restricted game $\left(N, \overline{u_{S}}\right)$ is $\mathcal{F}$-convex, then for every induced cycle of $G, C=\left(1, e_{1}, 2, e_{2}, \ldots\right.$, $\left.m, e_{m}, 1\right)$ with $m \geq 4$, the edge-weights satisfy, after renumbering the edges if necessary:

$$
u_{1} \leq u_{2} \leq u_{3}=\cdots=u_{m} .
$$

Moreover, if we consider the correspondence $\mathcal{P}_{G}$ and if we have also inheritance of superaddivity then:

$$
u_{1}=u_{2}=u_{3}=\cdots=u_{m},
$$

or

$$
u_{1}=u_{2}<\frac{u_{3}}{2}<u_{3}=\cdots=u_{m}
$$

Proof. Let us define $M:=\max _{1 \leq i \leq m} u_{i}$. Let us consider a maximal connected subset $A \subseteq C$ such that for all $e \in E(A), u(e)=M$. If $|C \backslash A| \leq 1$ the result is obviously satisfied. Let us assume $|C \backslash A| \geq 2$. After renumbering if necessary, we may assume $A=\{2,3, \ldots, l+1\}$, with $2 \leq l \leq m-2$, $e_{i}=\{i, i+1\}$ for $2 \leq i \leq l, e_{1}=\{1,2\}$, and $e_{l+1}=\{l+1, l+2\}$ as represented in Figure 5 for $l=3$. As $A$ is maximal we have $u_{1}, u_{l+1}<M$. Let us consider $B=A \cup\{l+2\}$ and $i=1$. Then we have $\mathcal{P}(A \cup\{i\})=\{A,\{i\}\}$ and $\mathcal{P}(B)=$ $\{A,\{l+2\}\}$. Therefore $\mathcal{P}(B)_{\mid A}=A \neq \mathcal{P}(A)=\{\{2\},\{3\}, \ldots,\{l+1\}\}$ and this contradicts Theorem 8 .

Let us now assume that there is also inheritance of superadditivity. Then 


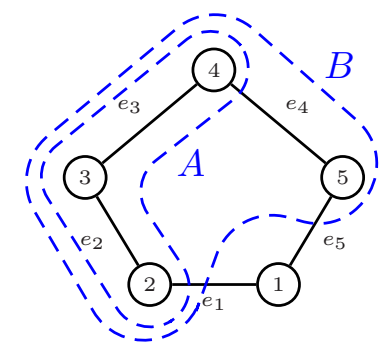

Figure 5:

by Theorem 1, the following condition is satisfied:

$$
\mathcal{P}(A) \text { is a refinement of } \mathcal{P}(B)_{\mid A}, \forall A \subseteq B \subseteq N .
$$

Let us consider $\sigma(C)=\min _{0 \leq k \leq m-2}\left(\frac{u_{1}+u_{2}+k M}{1+k}\right)$. Suppose first $u_{1}+u_{2} \geq$ $M$. Then, $\sigma(C)$ corresponds to $k=m-2$, and therefore $\mathcal{P}_{\max }(C)$ is obtained by deleting all edges, i.e., $\mathcal{P}_{\max }(C)=\{\{1\},\{2\}, \ldots,\{m\}\}$. Then condition (27) applied to $A=\{1,2,3,4\}$ and $B=C$ implies $u_{1}=u_{2}=M$. Suppose now $u_{1}+u_{2}<M$. Then, $\sigma(C)$ is obtained for $k=0$. Therefore $\mathcal{P}_{\max }(C)$ is obtained by deleting $e_{1}$ and $e_{2}$, i.e., $\mathcal{P}_{\max }(C)=\{\{2\}, C \backslash\{2\}\}$ and condition (27) implies $u_{1}=u_{2}$. Finally, as $u_{1}+u_{2}<M$, we get $u_{1}=u_{2}<\frac{M}{2}$.

We end this section with a counterexample to the inheritance of convexity. Let us consider the situation represented in Figure 6. We suppose:

$$
u_{2}=u_{3}<u_{4}<u_{1} .
$$

We define $A_{1}=B_{1}=\left\{v_{1}, v_{2}\right\}, A_{2}=\left\{v_{5}\right\}, B_{2}=\left\{v_{4}, v_{5}\right\}, A=A_{1} \cup A_{2}$ and $B=B_{1} \cup B_{2}$. According to (28), we have $u_{2}=u_{3}<\sigma\left(B_{2}\right)=u_{4}<\sigma\left(A_{1}\right)=$

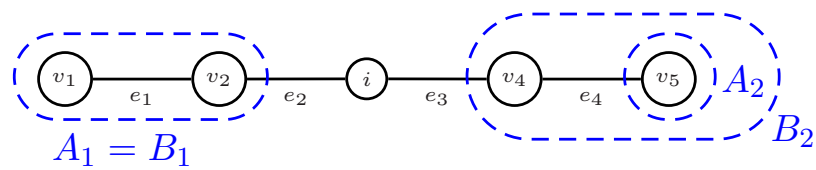

Figure 6:

$\sigma\left(B_{1}\right)=u_{1}$. Therefore $\mathcal{P}(B)=\left\{B_{1},\left\{v_{4}\right\},\left\{v_{5}\right\}\right\}, \mathcal{P}(A \cup\{i\})=\left\{A_{1}, A_{2},\{i\}\right\}$, and $\mathcal{P}(A)=\left\{\left\{v_{1}\right\},\left\{v_{2}\right\}, A_{2}\right\}$. Thus we have $A_{1} \in \mathcal{P}(A \cup\{i\})$ but $\mathcal{P}(B)_{\mid A_{1}}=$ $A_{1} \neq \mathcal{P}(A)_{\mid A_{1}}$. Then Theorem 8 proves there is no inheritance of convexity even if the condition of Proposition 9 is satisfied. This counterexample shows that the preceding conditions on edge-weights have no chance to be sufficient if we don't consider connected subsets and therefore the $\mathcal{F}$-convexity.

\subsection{Sufficient conditions}

Let $\mathcal{F}$ be the family of connected subsets of $N$. Henceforth $G$ will be a cyclefree graph. Therefore $\mathcal{P}_{\text {min }}=\mathcal{P}_{G}$. We will now prove that the preceding necessary conditions are also sufficient in this case for superadditive games. 
Theorem 12. Let $G=(N, E, u)$ be a cycle-free weighted graph. Let us consider the correspondence $\mathcal{P}=\mathcal{P}_{\min }$. For every superadditive and $\mathcal{F}$ convex game $(N, v)$, the restricted game $(N, \bar{v})$ is $\mathcal{F}$-convex if and only if the following conditions are satisfied:

1) (Convexity condition) For all paths $\gamma=\left\{e_{1}, e_{2}, \ldots, e_{m}\right\}$ in $G$ and for all $i, j, k$ such that $1 \leq i<j<k \leq m$, we have $u_{j} \leq \max \left(u_{i}, u_{k}\right)$.

2) (Branching condition) For all stars $S_{n}, n \geq 3$, with edges $e_{1}, e_{2}, \ldots, e_{n}$, the weights satisfy $u_{1} \leq u_{2}=u_{3}=\ldots=u_{n}$ after renumbering the edges if necessary.

Corollary 13. Let $G=(N, E, u)$ be a cycle-free weighted graph. If we consider on $G$ the correspondence $\mathcal{P}=\mathcal{P}_{\min }$, then the following properties are equivalent:

1) For each unanimity game $\left(N, u_{S}\right)$, the restricted game $\left(N, \overline{u_{S}}\right)$ is $\mathcal{F}$ convex.

2) For each superadditive and $\mathcal{F}$-convex game $(N, v)$, the restricted game $(N, \bar{v})$ is $\mathcal{F}$-convex.

3) For all $A, B \in \mathcal{F}$ such that $A \cap B \in \mathcal{F}, \mathcal{P}(A \cap B)=\left\{A_{j} \cap B_{k} ; A_{j} \in\right.$ $\mathcal{P}(A), B_{k} \in \mathcal{P}(B)$ s.t. $\left.A_{j} \cap B_{k} \neq \emptyset\right\}$.

4) For all $i \in N$, for all $A \subset B \subseteq N \backslash\{i\}$ with $A, B, A \cup\{i\} \in \mathcal{F}$, and for all $A^{\prime} \in \mathcal{P}(A \cup\{i\}), \mathcal{P}(B)_{\mid A^{\prime}}=\mathcal{P}(A)_{\mid A^{\prime}}$.

Proof. Let us assume 1) is satisfied. Then Propositions 9 and 10 imply that the conditions of Theorem 12 are satisfied and therefore 2) is satisfied. Obviously 2) implies 1 ). As we consider the correspondence $\mathcal{P}_{\min }$, Corollary 2 implies that we have inheritance of superadditivity. Then by Theorem 8,1 ) is equivalent to 3 ) and 4).

Before proving Theorem 12, we establish some useful lemmas.

Lemma 14. Let us consider subsets $A, B \subseteq N$ and a partition $\left\{B_{1}, B_{2}, \ldots\right.$, $\left.B_{p}\right\}$ of $B$. If $A, B_{i}$, and $A \cap B_{i} \in \mathcal{F}$, for all $i \in\{1, \ldots, p\}$, then for every $\mathcal{F}$-convex game $(N, v)$ we have:

$$
v(A \cup B)+\sum_{i=1}^{p} v\left(A \cap B_{i}\right) \geq v(A)+\sum_{i=1}^{p} v\left(B_{i}\right) .
$$

Proof. We prove the result by induction. (29) is obviously satisfied for $p=1$. Let us assume it is satisfied for $p$ and let us consider a partition 
$\left\{B_{1}, B_{2}, \ldots, B_{p}, B_{p+1}\right\}$ of $B$. We set $B^{\prime}=B_{1} \cup B_{2} \cup \ldots \cup B_{p}$. The $\mathcal{F}$ convexity of $v$ applied to the subsets $A \cup B^{\prime}$ and $B_{p+1}$ provides the following inequality:

$$
v\left(\left(A \cup B^{\prime}\right) \cup B_{p+1}\right)+v\left(\left(A \cup B^{\prime}\right) \cap B_{p+1}\right) \geq v\left(A \cup B^{\prime}\right)+v\left(B_{p+1}\right) .
$$

By induction (29) is valid for $B^{\prime}$ :

$$
v\left(A \cup B^{\prime}\right)+\sum_{i=1}^{p} v\left(A \cap B_{i}\right) \geq v(A)+\sum_{i=1}^{p} v\left(B_{i}\right) .
$$

Adding (30) and (31) we obtain the result for $p+1$.

Lemma 15. Let us consider a correspondence $\mathcal{P}$ on $N$ and subsets $A \subseteq$ $B \subseteq N$ such that $\mathcal{P}(A)=\mathcal{P}(B)_{\mid A}$. If $A \in \mathcal{F}$ and if all elements of $\mathcal{P}(A)$ and $\mathcal{P}(B)$ are in $\mathcal{F}$, then for every $\mathcal{F}$-convex game $(N, v)$ we have:

$$
v(B)-\bar{v}(B) \geq v(A)-\bar{v}(A) .
$$

Proof. If $\mathcal{P}(B)=\left\{B_{1}, B_{2}, \ldots, B_{p}\right\}$ then $\mathcal{P}(A)=\left\{A \cap B_{1}, A \cap B_{2}, \ldots, A \cap B_{p}\right\}$, and Lemma 14 implies (32).

The following lemma gives a simple condition ensuring $\mathcal{P}_{\min }(A)$ is induced by $\mathcal{P}_{\min }(B)$ for $A \subseteq B$.

Lemma 16. Let $G=(N, E, u)$ be a cycle-free weighted graph and let us consider $A \subseteq B \subseteq N$ such that $\sigma(A)=\sigma(B)$. Then $\mathcal{P}_{\min }(A)=\mathcal{P}_{\min }(B)_{\mid A}$. Moreover if $A \in \mathcal{F}$ then for every $\mathcal{F}$-convex game $(N, v)$ we have:

$$
v(B)-\bar{v}(B) \geq v(A)-\bar{v}(A) .
$$

Proof. We have to prove for every component $B_{k}$ of $\mathcal{P}_{\min }(B)$ with $B_{k} \cap A \neq \emptyset$, that $B_{k} \cap A$ is a component $A_{k}$ of $\mathcal{P}_{\min }(A)$. Let $\alpha_{0}$ be a fixed vertex of $A \cap B_{k}$ and $A_{k}$ be the component of $\mathcal{P}_{\min }(A)$ which contains $\alpha_{0}$. We will prove $A \cap B_{k}=A_{k}$. As $\sigma(A)=\sigma(B), \Sigma(A)=E(A) \cap \Sigma(B)$. Let now $\alpha_{1}$ be another vertex of $A_{k}$ and $\gamma$ be a path in $A_{k}$ from $\alpha_{0}$ to $\alpha_{1}$. Each edge $e$ of $\gamma$ is in $E(A) \backslash \Sigma(A)$ and therefore satisfies $u(e)>\sigma(A)$. As $\sigma(A)=\sigma(B)$ and $A \subseteq B$, each edge $e$ of $\gamma$ is in $E(B)$ and satisfies $u(e)>\sigma(B)$, i.e., $e \in E(B) \backslash \Sigma(B)$. Therefore $\gamma$ is a path from $\alpha_{0}$ to $\alpha_{1}$ in $B$ and therefore $\alpha_{1} \in B_{k}$. That is :

$$
A_{k} \subseteq A \cap B_{k} .
$$

Let now $\alpha_{1}$ be a vertex in $A \cap B_{k}$. As $A$ is connected, there exists a path $\gamma$ from $\alpha_{0}$ to $\alpha_{1}$ in $A$ and possibly another one $\gamma^{\prime}$ from $\alpha_{0}$ to $\alpha_{1}$ in $B_{k}$. But as $G$ has no cycle $\gamma=\gamma^{\prime}$ and $\gamma$ is a path in $A \cap B_{k}$. For each edge $e$ of $\gamma, e$ is in $E(A)$ and in $E(B) \backslash \Sigma(B)$, that is $u(e)>\sigma(B)$. As $\sigma(A)=\sigma(B)$, we 
have also $u(e)>\sigma(A)$ and therefore $e \in E(A) \backslash \Sigma(A)$. Thus $\gamma$ is a path of a component of $\mathcal{P}_{\min }(A)$. As $\alpha_{0} \in A_{k}, \gamma$ is a path in $A_{k}$ and then $\alpha_{1} \in A_{k}$. That is:

$$
A \cap B_{k} \subseteq A_{k}
$$

Following (34) and (35), we have shown $A \cap B_{k}=A_{k}$. Lemma 15 implies (33).

Lemma 17. Let $G=(N, E, u)$ be a cycle-free weighted graph. Let us assume that the edge-weight function $u$ satisfies the convexity conditions 1) and 2) of Theorem 12. If $A$ and $B$ are connected, $A \subseteq B \subseteq N \backslash\{i\}, j \in A \cap B$ and $e:=\{i, j\} \in E$, then either $u(e) \geq \sigma(A) \geq \sigma(B)$ or $\sigma(A)=\sigma(B)>u(e)$.

Proof. As $A \subseteq B$, we have $\sigma(A) \geq \sigma(B)$. Let us assume:

$$
\sigma(A)>u(e)
$$

As $A$ and $B$ are connected, we can consider a path $\gamma_{1}=\left(e_{1}, e_{2}, \ldots, e_{m}\right)$ in $G_{A}$ such that $u\left(e_{1}\right)=\sigma(A)$ and $j$ is an end-vertex of $e_{m}$, and a path $\gamma_{2}=\left(e_{1}^{\prime}, e_{2}^{\prime}, \ldots, e_{r}^{\prime}\right)$ in $G_{B}$ such that $u\left(e_{1}^{\prime}\right)=\sigma(B)$ and $j$ is an end-vertex of $e_{r}^{\prime}$, as represented in Figure 7 . The convexity condition applied to the

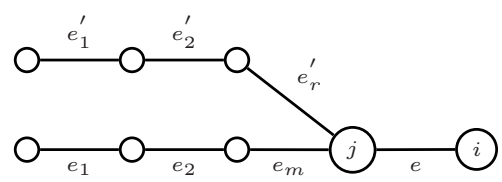

Figure 7:

path $\gamma_{1} \cup\{e\}$ and (36) imply $u\left(e_{m}\right) \leq \max \left(u\left(e_{1}\right), u(e)\right)=u\left(e_{1}\right)=\sigma(A)$. As $e_{m} \in E(A), u\left(e_{m}\right)=\sigma(A)$ and using again (36), we obtain:

$$
u\left(e_{m}\right)>u(e) .
$$

If $e_{m}=e_{r}^{\prime}$, we have $u\left(e_{r}^{\prime}\right)=u\left(e_{m}\right)=\sigma(A)$. If now $e_{m} \neq e_{r}^{\prime}$, the branching condition for the three edges $e_{m}, e_{r}^{\prime}, e$ and (37) imply again $u\left(e_{m}\right)=u\left(e_{r}^{\prime}\right)=$ $\sigma(A)$. The convexity condition applied to the path $\gamma_{2} \cup\{e\}$ imply $u\left(e_{r}^{\prime}\right) \leq$ $\max \left(u\left(e_{1}^{\prime}\right), u(e)\right)$ and therefore:

$$
\sigma(A) \leq \max (\sigma(B), u(e)) .
$$

Then (36) and (38) imply $\sigma(A) \leq \sigma(B)$. Therefore we have $\sigma(A)=\sigma(B)>$ $u(e)$. 
Proof of Theorem 12. We have already seen in Section 5.1 that conditions 1 and 2 are necessary. We now prove they are sufficient. Let $(N, v)$ be a given $\mathcal{F}$-convex game. According to Theorem 4 , we have to prove that, for all $i \in N$, for all $A \subseteq B \subseteq N \backslash\{i\}$ and $A, B, A \cup\{i\} \in \mathcal{F}$, we have:

$$
\bar{v}(B \cup\{i\})-\bar{v}(B) \geq \bar{v}(A \cup\{i\})-\bar{v}(A) .
$$

We will consider $\mathcal{P}(A)=\left\{A_{1}, A_{2}, \ldots, A_{p}\right\}$ and $P(B)=\left\{B_{1}, B_{2}, \ldots, B_{q}\right\}$. As $A \cup\{i\}$ is connected, there exists an edge $e=\{i, j\}$ with $j \in A$ (and therefore $j \in A \cap B$ ). As $A$ is connected and as $G$ is cycle-free, $e$ is necessarily unique. Using Lemma 17, we have several cases to consider.

Case $1 \sigma(A)=\sigma(B)>u(e)$. Then $\mathcal{P}_{\min }(A \cup\{i\})=\{A,\{i\}\}, \mathcal{P}_{\min }(B \cup$ $\{i\})=\{B,\{i\}\}$, and therefore $\bar{v}(A \cup\{i\})=v(A)+v(i)$ and $\bar{v}(B \cup\{i\})=$ $v(B)+v(i)$. Then (39) becomes equivalent to $v(B)-\bar{v}(B) \geq v(A)-\bar{v}(A)$. As $\sigma(A)=\sigma(B)$, Lemma 16 implies that this last inequality is satisfied.

Case $2 u(e)=\sigma(A) \geq \sigma(B)$. Then $\mathcal{P}_{\min }(A \cup\{i\})=\left\{\mathcal{P}_{\min }(A),\{i\}\right\}$ and $\bar{v}(A \cup\{i\})=\bar{v}(A)+v(i)$. According to Corollary 2, $\bar{v}$ is superadditive. Therefore $\bar{v}(B \cup\{i\})-\bar{v}(B) \geq \bar{v}(i)=v(i)=\bar{v}(A \cup\{i\})-\bar{v}(A)$.

Case $3 u(e)>\sigma(A) \geq \sigma(B)$. Let us suppose w.l.o.g. $j \in A_{1} \cap B_{1}$. Then $\mathcal{P}(A \cup\{i\})=\left\{A_{1} \cup\{i\}, A_{2}, \ldots, A_{p}\right\}$ and $\mathcal{P}(B \cup\{i\})=\left\{B_{1} \cup\{i\}, B_{2}, \ldots, B_{q}\right\}$.

We obtain:

$$
\bar{v}(A \cup\{i\})-\bar{v}(A)=v\left(A_{1} \cup\{i\}\right)-v\left(A_{1}\right),
$$

and

$$
\bar{v}(B \cup\{i\})-\bar{v}(B)=v\left(B_{1} \cup\{i\}\right)-v\left(B_{1}\right) .
$$

As $A \subseteq B$ and as $G$ is a cycle-free graph, we have $\Sigma(B) \cap E(A)=\emptyset$ or $\Sigma(B) \cap E(A)=\Sigma(A)$. Therefore $A_{1} \subseteq B_{1}$ and by $\mathcal{F}$-convexity of $(N, v)$ we have:

$$
v\left(B_{1} \cup\{i\}\right)-v\left(B_{1}\right) \geq v\left(A_{1} \cup\{i\}\right)-v\left(A_{1}\right) .
$$

Using (40) and (41), (42) is equivalent to (39).

Let us observe that, as $G$ has no cycle, if $v$ is superadditive, $\bar{v}$ is superadditive using Corollary 2. Hence if $A$ and $B$ are connected and $A \cap B=\emptyset$ we still have $\bar{v}(A \cup B) \geq \bar{v}(A)+\bar{v}(B)$. For all connected subsets $A$ and $B$ of $N$, we have $\bar{v}(A \cup B)+\bar{v}(A \cap B) \geq \bar{v}(A)+\bar{v}(B)$ (assuming $v$ is $\mathcal{F}$-convex and superadditive).

\section{Shapley value}

We now investigate the computation of the Shapley value of the game $\bar{v}$ in the case of cycle-free graphs. We assume that $G=(N, E)$ is a tree 
(results easily extend to forests). To compute the Shapley value, we have to compute $\bar{v}(S \cup i)-\bar{v}(S)$ for every $S \subseteq N \backslash i$ and every $i \in N$. Let us consider some fixed $i$ and $S$. Let $S_{1}, \ldots, S_{k}$ be the connected components of $S, k \geq 1$, and suppose that $i$ is linked to the components $S_{1}, \ldots, S_{l}$ and not to the others, with $l \in\{0,1, \ldots, k\}, l=0$ indicating that no component is linked to $i$. We remark that all cases are covered: $S$ and $S \cup i$ connected correspond to $k=l=1, S$ connected and $S \cup i$ not connected correspond to $k=1, l=0$, etc. Since $S_{1}, \ldots, S_{l}$ are connected to $i$, there exist edges $\left\{i, j_{1}\right\}, \ldots,\left\{i, j_{l}\right\}$ from $i$ to each $S_{1}, \ldots, S_{l}$, with weights $u_{1}, \ldots, u_{l}$, assuming w.l.o.g. $u_{1} \leq \cdots \leq u_{l}$, and $u_{0}$ is the minimal weight on $S$. Put $\mathcal{P}_{\min }\left(S_{\ell}\right)=$ $\left\{S_{\ell, 1}, \ldots, S_{\ell, p_{\ell}}\right\}, \ell=1, \ldots, k$ with $S_{\ell, 1} \ni j_{\ell}$ for $\ell=1, \ldots, l$. Then

(43) $\bar{v}(S \cup i)-\bar{v}(S)$

$$
=\left\{\begin{array}{l}
v\left(S_{1,1} \cup \cdots \cup S_{l, 1} \cup i\right)-v\left(S_{1,1}\right)-\cdots-v\left(S_{l, 1}\right) \\
\quad \text { if } u_{0}<u_{1} \\
\sum_{r=1}^{m} v\left(S_{r}\right)+v\left(S_{m+1} \cup \cdots \cup S_{l} \cup i\right)-\sum_{r=1}^{l} \sum_{s=1}^{p_{r}} v\left(S_{r, s}\right) \\
\quad \text { if } u_{1}=\cdots=u_{m}<\min \left(u_{0}, u_{m+1}\right) \\
v\left(S_{m+1,1} \cup \cdots \cup S_{l, 1} \cup i\right)-v\left(S_{m+1,1}\right)-\cdots-v\left(S_{l, 1}\right) \\
\quad \text { if } u_{1}=\cdots=u_{m}=u_{0}<u_{m+1} .
\end{array}\right.
$$

Note that $m=l$ is allowed, in which case $S_{m+1} \cup \cdots \cup S_{l}=\emptyset$ and $S_{m+1,1} \cup$ $\cdots \cup S_{l, 1}=\emptyset$.

The above formula, although complicated, gives an explicit expression of $\bar{v}(S \cup i)-\bar{v}(S)$ for all $S, i$. Another way of computing the Shapley value is to compute $\bar{v}$ iteratively using a suitable ordering of the players, say, $i_{1}, i_{2}, \ldots, i_{n}$, for which the induced subgraph of the players $i_{1}, \ldots, i_{k}$ is an extension of the one of $i_{1}, \ldots, i_{k-1}$ by at most one edge. Such an ordering can be produced by the following algorithm:

\section{SEQUENCING ALGORITHM}

Init: $N$ set of nodes, $i \in N . L \leftarrow\{i\}, N \leftarrow N \backslash\{i\}$.

Do Until $N=\emptyset$ :

- Choose $j \in N$ which is neighbor of some $i \in L$

$$
\text { - } L \leftarrow L \cup\{j\}, N \leftarrow N \backslash\{j\}
$$

\section{End do}

We claim that at each step, only one edge is added to the subgraph. Indeed, at each step the subgraph induced by $L$ is connected, and being a subgraph of $N$, is a tree. Then any new node $j$ has exactly one link in $L$, otherwise there would exist a cycle in $L$. 
Supposing that the sequence $i_{1}, i_{2}, \ldots, i_{n}$ is built and that all values $\bar{v}(S)$ with $S \subseteq\left\{i_{1}, \ldots, i_{k-1}\right\}$ have been computed, the computation of $\bar{v}\left(S \cup i_{k}\right)$ is done as follows:

$$
\bar{v}\left(S \cup i_{k}\right)= \begin{cases}v(S)+v(i), & \text { if } u(e)<u_{o} \\ v\left(S_{1} \cup i\right)+v\left(S_{2}\right)+\cdots+v\left(S_{l}\right), & \text { if } u(e)>u_{0} \\ \bar{v}(S)+v(i), & \text { if } u(e)=u_{0},\end{cases}
$$

with, as before, $u_{0}$ the minimal weight in $S, \mathcal{P}_{\min }(S)=\left\{S_{1}, \ldots, S_{l}\right\}$, and $e$ the new edge formed, linking $i$ to $S_{1}$.

We finish this section by giving a property which may considerably simplify the calculus of the Shapley value. A player $i$ is called a dummy player in a game $(N, v)$ if $v(S \cup\{i\})=v(S)+v(\{i\})$ for every $S \subseteq N \backslash i$.

Proposition 18. If there exists a dummy player $i$ for $\bar{v}$, then either $\{i\}$ has degree at most 1 , or all players are dummy for $\bar{v}$.

Proof. Suppose $i$ is dummy for $\bar{v}$. Then for any $S \subseteq N \backslash i$,

$$
\bar{v}(S \cup\{i\})=\bar{v}(S)+\bar{v}(\{i\})=\bar{v}(S)+v(\{i\}) .
$$

According to (43), this happens if there is no edge from $i$ to $S(l=0)$. Therefore, one possibility is that $i$ has no edge, i.e., it has degree 0. Suppose then that this is not the case. Then there exist, say $m$ edges adjacent to $i$, denoted by $\left\{i, j_{1}\right\}, \ldots,\left\{i, j_{m}\right\}$ with weight $u_{1}, \ldots, u_{m}$. Only the 3 d equation in (43) with $m=l$ permits to get $\bar{v}(S \cup i)-\bar{v}(S)=v(i)$, which means that $u_{1}=u_{2}=\cdots=u_{m}=\min _{e \in E(S)} u(e)$.

Claim: any edge in the graph has same weight $u_{1}$ if $m>1$.

Indeed, take any edge $e=\{k, l\}$ in $E$. If $k$ or $l=i$, then we know already that $u(e)=u_{1}$. Suppose now there exists $p \in\{1, \ldots, m\}$ such that $k$ or $l=j_{p}$ and consider $S=\{k, l\}$. Then imposing (44) for $S$ shows that $u(e)=u_{1}$ is the only possibility. Suppose finally that both $k, l$ differ from $i, j_{1}, \ldots, j_{m}$. If there is no edge linking $j_{1}$ to $k$ or $l$, let us take $S=\left\{j_{1}, k, l\right\}$. The partition of $S$ is the partition in singletons since $\{k, l\}$ is not connected with $j_{1}$. Therefore to satisfy (44), the partition of $S \cup\{i\}$ must also be in singletons, which implies that $u(e)=u_{1}$. If there is an edge between $j_{1}$ and $k$ or $l$, then, as the graph is cycle free, there is no edge linking $k$ or $l$ to $j_{p}$ for $p \in\{2, \ldots, m\}$. Taking now $S=\left\{j_{2}, k, l\right\}$ we still get $u(e)=u_{1}$.

Now if all weights are equal, $\bar{v}$ is additive, therefore all players are dummy.

\section{$7 \quad$ A second family of restricted games associated with the strength of a graph}

Let $(N, v)$ be a game on the set $N$ of vertices of the graph $G=(N, E)$ and let $u: E \rightarrow \mathbb{R}^{+}$be a weight function on the set of edges. For a family $\mathcal{F}$ 
of subsets of $N$ and a subset $A$ of $N$, we denote by $\mathcal{F}(A)$ the elements of $\mathcal{F}$ included in $A$.

$$
\mathcal{F}(A):=\{F \in \mathcal{F} ; F \subseteq A\} .
$$

We consider on the set of players $N$ a hierarchy of coalition structures, that is, a finite number of partitions $\mathcal{P}_{0}, \mathcal{P}_{1}, \ldots, \mathcal{P}_{m}$ of $N$ such that $\mathcal{P}_{0}=\{N\}$, $\mathcal{P}_{m}=\{\{1\},\{2\}, \ldots,\{n\}\}$ is the singleton coalition structure, and:

$$
\mathcal{P}_{m} \leq \mathcal{P}_{m-1} \leq \cdots \leq \mathcal{P}_{i+1} \leq \mathcal{P}_{i} \leq \cdots \leq \mathcal{P}_{1} \leq \mathcal{P}_{0}
$$

where $\mathcal{P}_{i+1} \leq \mathcal{P}_{i}$ means that every block of $\mathcal{P}_{i+1}$ is a subset of a block of $\mathcal{P}_{i}$. $\mathcal{P}_{1}$ is one of the partitions of $N$ given by the strength of the graph $G$. For every $A \in \mathcal{P}_{i}$, we consider the subgraph $G_{A}=(A, E(A))$. We select a minimizer of $\sigma\left(G_{A}\right)$ and consider the corresponding partition of $A$. This partition provides the blocks of $\mathcal{P}_{i+1}$ which are subsets of $A$. Let $\mathcal{F}$ be the family

$$
\mathcal{F}=\mathcal{P}_{0} \cup \mathcal{P}_{1} \cup \ldots \cup \mathcal{P}_{m}
$$

We define a new game $(N, \tilde{v})$ by:

$$
\tilde{v}(A):=\sum_{F \in \mathcal{F}(A), F \text { maximal }} v(F) \quad \text { for all } A \in N .
$$

The family of sets $\mathcal{F}$ obviously satisfies the following property. For all $A, B \in$ $\mathcal{F}$, one and only one of the following properties is verified: $A \cap B=\emptyset$, or $A \subset B$ or $B \subset A$ or $A=B$. We say that $\mathcal{F}$ is a nested family. Therefore $\mathcal{F}$ is also an intersecting system ${ }^{2}, \mathcal{F}$ is also weakly union-closed ${ }^{3}$ and therefore $\mathcal{F} \cup \emptyset$ is also a partition system ${ }^{4}$ (cf. $[1,5,8]$ ).

We recall that a game $(N, v)$ is convex if the function $v$ is supermodular i.e., for all $A, B \in 2^{N}, v(A \cup B)+v(A \cap B) \geq v(A)+v(B)$. We say $v$ is $\mathcal{F}$-superadditive if for all $A, B \in \mathcal{F}$ such that $A \cap B=\emptyset$, we have:

$$
v(A \cup B) \geq v(A)+v(B) .
$$

Observe that for such games, since $\mathcal{F}$ is a nested family, we have for all $A, B \in \mathcal{F}:$

$$
v(A \cup B)+v(A \cap B) \geq v(A)+v(B) .
$$

A game $v$ defined on an intersecting system $(N, \mathcal{F})$ and satisfying condition (50) is called an intersecting convex game (cf. [5]). Algaba et al. [1] and Faigle [5] have proved:

\footnotetext{
${ }^{2}$ If $A$ and $B \in \mathcal{F}$ and if $A \cap B \neq \emptyset$ then $A \cap B$ and $A \cup B$ are in $\mathcal{F}$.

${ }^{3}$ If $A$ and $B$ are in $\mathcal{F}$ and if $A \cap B \neq \emptyset$ then $A \cup B \in \mathcal{F}$.

${ }^{4}$ For all $A \in \mathcal{F}$, the maximal subsets $F \in \mathcal{F}(A)$ form a partition of $A$, and the singletons are in $\mathcal{F}$.
} 
Theorem 19. If $(N, \mathcal{F}, v)$ is an intersecting convex game, then the restricted game $\left(N, 2^{N}, \tilde{v}\right)$ defined by:

$$
\tilde{v}(A)=\sum_{F \in \mathcal{F}(A), F \text { maximal }} v(F)
$$

for all $A \subseteq N$, is a convex game.

This last theorem applies to any preceding nested family $\mathcal{F}$ we have constructed by (45), (46), (47) and (48) using the strength of a graph, and therefore the following theorem is a corollary of Theorem 19:

Theorem 20. If $\mathcal{F}$ is a family of subsets of $N$ associated with the strength of a graph $G(N, E)$ by means of the preceding hierarchy of coalitions structures, and if $(N, \mathcal{F}, v)$ is an $\mathcal{F}$-superadditive game on $N$, then the restricted game $\left(N, 2^{N}, \tilde{v}\right)$ is a convex game.

We give in Appendix A a direct proof following the method of A. Van den Nouweland and P. Borm (1991) [14].

\section{Conclusion}

All preceding games are point games on the set $N$ of vertices of $G$. Borm, Owen and Tijs in 1990 [3] have introduced arc games on the set $E$ of edges of $G$ and the position value. We could also consider arc games in the same spirit, by substituting to the partition into connected components the partition associated with the strength of the graph. Aziz et al. [2] have investigated some properties of the wiretapping game associated with a given graph. The value of this game is precisely equal to the reciprocal of the strength of the graph. Using the strength of the subgraphs they construct a prime partition of the set of edges which is of main interest to analyse the wiretapping game. By means of this prime partition we could also construct for a given arc game $v$ on $E$ a new restricted game. It would be interesting to study inheritance of superadditivity and convexity for this type of games.

\section{References}

[1] E. Algaba, J.M. Bilbao, and J.J. Lopez. A Unified Approach to Restricted Games. Theory and Decision, 50(4):333-345, 2001.

[2] Haris Aziz, Oded Lachish, Mike Paterson, and Rahul Savani. Wiretapping a Hidden Network. In Proceedings of the 5th International Workshop on Internet and Network Economics, WINE '09, pages 438-446, Berlin, Heidelberg, 2009. Springer-Verlag. 
[3] P. Borm, G. Owen, and S. Tijs. Values of points and arcs in communication situations. Technical Report 9004, Dept of Mathematics, University of Nijmegen, The Netherlands, 1990.

[4] W.H. Cunningham. Optimal attack and reinforcement of a network J. ACM, 32(3):549-561, 1985.

[5] U. Faigle. Cores of Games with Restricted Cooperation. ZOR - Methods and Models of Operations Research, 33(6):405-422, 1989.

[6] J. Fonlupt and A. Skoda. Strongly Polynomial Algorithm for the Intersection of a Line with a Polymatroid In Research Trends in Combinatorial Optimization, pages 69-85. Springer, 2008.

[7] S. Fujishige. Submodular Functions and Optimization, volume 58 of Annals of Discrete Mathematics. Elsevier, second edition, 2005.

[8] M. Grabisch. The Core of Games on Ordered Structures and Graphs. 4OR: A Quarterly Journal of Operations Research, 7(3):207-238, 2009.

[9] D. Gusfield. Computing the strength of a graph. SIAM J. Comput., 20(4):639-654, 1991.

[10] R.B. Myerson. Graphs and Cooperation in Games. Mathematics of Operations Research, 2(3):225-229, 1977.

[11] G. Owen. Values of graph-restricted games. SIAM J. Algebraic Discrete Methods, 7(2):210-220, 1986.

[12] A. Schrijver. Combinatorial Optimization : Polyhedra and Efficiency. Springer-Verlag, 2003.

[13] A. Skoda. A new Algorithm for the intersection of a line with the independent set polytope of a matroid. Bulletin des Sciences Mathématiques, 133(2):169-185, 2009.

[14] A. van den Nouweland and P. Borm. On the Convexity of Communication Games. International Journal of Game Theory, 19(4): 421-30, 1991. 


\section{A Proof of Theorem 20}

We give a direct proof following the method of A. Van den Nouweland and P. Borm (1991) [14].

Proof. For all $A, B \in N$ such that $A \cap B=\emptyset$, we have $v(A \cup B) \geq v(A)+v(B)$. Let us now consider $A, B$ and $i \in N$ such that $A \subset B \subseteq N \backslash\{i\}$. We have to prove $\tilde{v}(A \cup\{i\})-\tilde{v}(A) \leq \tilde{v}(B \cup\{i\})-\tilde{v}(B)$. By definition, we have:

$$
\tilde{v}(A)=\sum_{C \in \mathcal{F}(A), C \text { maximal }} v(C)
$$

and

$$
\tilde{v}(A \cup\{i\})=\sum_{C \in \mathcal{F}(A \cup\{i\}), C \text { maximal }} v(C) .
$$

Let us denote by $C(i)$ the unique maximal set $C \in \mathcal{F}(A \cup\{i\})$ such that $i \in C$. Let us denote by $\mathcal{C}$ the family:

$$
\mathcal{C}:=\{C \in \mathcal{F}(A), C \text { maximal in } \mathcal{F}(A) \text { and } C \subset C(i)\} .
$$

Observe that as $i \notin A, C(i)=\{i\} \cup\left(\bigcup_{C \in \mathcal{C}} C\right.$ ) (If $C \in \mathcal{F}, C \subset A \cup\{i\}, C$ is maximal in $\mathcal{F}(A \cup\{i\})$ and $i \notin C$, then $C \subset A$ and $C$ is maximal in $\mathcal{F}(A)$.) Observe also that if $C \in \mathcal{F}(A \cup\{i\}), C$ is maximal and $C \not \subset C(i)$ then $C \cap C(i)=\emptyset$ (partition) and $C \in \mathcal{F}(A)$ with $C$ maximal in $\mathcal{F}(A)$. Hence:

$$
\tilde{v}(A \cup\{i\})-\tilde{v}(A)=v(C(i))-\sum_{C \in \mathcal{C}} v(C) .
$$

Analogously, we define $D(i)$ as the maximal set $D$ in $\mathcal{F}(B \cup\{i\})$ such that $i \in D$ and :

$$
\mathcal{D}:=\{D \in \mathcal{F}(B) ; D \text { maximal in } \mathcal{F}(B), D \subset D(i)\} .
$$

Then $D(i)=\{i\} \cup\left(\bigcup_{D \in \mathcal{D}} D\right)$ and :

$$
\tilde{v}(B \cup\{i\})-\tilde{v}(B)=v(D(i))-\sum_{D \in \mathcal{D}} v(D) .
$$

Hence, it remains to prove that:

$$
v(C(i))-\sum_{C \in \mathcal{C}} v(C) \leq v(D(i))-\sum_{D \in \mathcal{D}} v(D) .
$$

We want now to prove that for every $C \in \mathcal{C}$, there exists one and only one $D \in \mathcal{D}$ such that $C=D$. As $A \subset B, A \cup\{i\} \subset B \cup\{i\}$ and therefore $C(i) \subseteq D(i)$. Hence, for all $C \in \mathcal{C}$, there exists precisely one $D \in \mathcal{D}$ 
such that $C \subseteq D . \quad D \cap C(i) \neq \emptyset$ because $D \cap C(i) \supset C \neq \emptyset . \quad D \supseteq C(i)$ contradicts $i \notin D$. Therefore $D \subset C(i)$. But $i \notin D$ and $C(i) \subset A \cup\{i\}$, then $D \subset A$. But $D$ is maximal in $\mathcal{F}(B)$, hence $D \subset A$ is maximal in $\mathcal{F}(A)$. As $C \subset D \subset A$ and $C$ and $D$ are maximal in $\mathcal{F}(A)$, we have $C=D$. We can now number the elements of $\mathcal{C}$ and $\mathcal{D}$ in such a way that $\mathcal{C}=\left\{C_{1}, C_{2}, \ldots, C_{s}\right\}, \mathcal{D}=\left\{D_{1}, D_{2}, \ldots, D_{t}\right\}$ with $s \leq t$ and $C_{r}=D_{r}$ for all $r, 1 \leq r \leq s$. Superadditivity of the game $(N, v)$ implies :

$$
v\left(\{i\} \cup \bigcup_{r=1}^{s} D_{r} \cup \bigcup_{r=s+1}^{t} D_{r}\right) \geq v\left(\{i\} \cup \bigcup_{r=1}^{s} D_{r}\right)+\left(\sum_{r=s+1}^{t} v\left(D_{r}\right)\right) .
$$

Then :

$$
v\left(\{i\} \cup \bigcup_{D \in \mathcal{D}} D\right)-\sum_{D \in \mathcal{D}} v(D) \geq v\left(\{i\} \cup \bigcup_{r=1}^{s} D_{r}\right)-\left(\sum_{r=1}^{s} v\left(D_{r}\right)\right) .
$$

As $D_{r}=C_{r}$ for all $r, 1 \leq r \leq s$, we obtain:

$$
\begin{aligned}
v\left(\{i\} \cup \bigcup_{D \in \mathcal{D}} D\right)-\sum_{D \in \mathcal{D}} v(D) & \geq v\left(\{i\} \cup \bigcup_{r=1}^{s} C_{r}\right)-\sum_{r=1}^{s} v\left(C_{r}\right) \\
& \geq v\left(\{i\} \cup \bigcup_{C \in \mathcal{C}} C\right)-\sum_{C \in \mathcal{C}} v(C)
\end{aligned}
$$

That is precisely (57). 